\title{
A LOGISTIC REGRESSION BASED HYBRID MODEL FOR BREAST CANCER CLASSIFICATION
}

\author{
Tina Elizabeth Mathew \\ Research Scholar, Technology Management, Department of Future Studies \\ University of Kerala, Thiruvananthapuram, 695581 Kerala, India \\ Email:tinamathew04@gmail.com \\ K S Anil Kumar \\ Associate Professor \& Guide, Technology Management, Department of Future Studies \\ University of Kerala, Thiruvananthapuram, 695581 Kerala, India \\ Email:ksanilksitm@gmail.com
}

\begin{abstract}
Data mining techniques are being used for breast cancer classification and good performance accuracy has been obtained while using the techniques individually or as ensembles. A notable problem is the skewed nature of the data which leads to imbalance among the output classes. The minority class being the negative class usually are smaller in number than the positive majority class. This usually leads to a moderate accuracy value for the classifier. The correct classification of minority cases is a significant problem. All classes need to be given equal importance during classification. In this study a hybrid model based on Logistic Regression is implemented with class balancing and ant search techniques and the performance is evaluated on the two class Wisconsin breast cancer dataset. A performance accuracy of $99.4 \%$ was obtained.
\end{abstract}

Keywords: Synthetic Minority Over-sampling Technique (SMOTE), Oversampling (OS), Random Undersampling (US), Neural Networks (NN), Random Forest (RF), Logistic Regression (LR), Support Vector Machines (SVM), Naïve Bayes (NB), Ant Search (AS)

\section{Introduction}

Breast Cancer is the most common and frequently diagnosed cancer in women worldwide and is the leading cause of death in women. Early detection is the key to survival and better breast outcome. Machine Learning techniques are widely used for disease diagnosis and outcome prediction [Durga et al., (2020), Kotsantis, (2007), Arafat et al.,(2012), Sivapriya et al..(2015), Mathew, (2019a), Mathew, (2019c)]. Classification is a machine learning technique that is used for producing class labels. In disease diagnosis, classifiers categorize the solution into respective classes based on the features space provided. In the training process, using the samples provided the classifier trains itself to produce the most optimal result. Once the model is constructed any new instance provided is expected to produce the finest result. The problem with the feature space in most cases is its large dimensionality. High number of attributes and large dimension of the feature space leads to the curse of dimensionality [Li et al., (2017)]. Feature selection, extraction and feature reduction helps to overcome this problem. It helps in identifying and extracting the most relevant information without loss of necessary information. Hence the primary goal of feature Search is to help in selecting relevant attributes [Rahman and Muniyandi, (2020), Rania and Ramyachitra, (2018), Jabbar, (2019), Sadhegipour et al., (2015)] Reduction of attributes and dimension helps in reducing the cost and improving accuracy by eliminating redundant or irrelevant information [Durga et al., (2020)]. Besides this, Feature search methods can also be used as classifiers for disease diagnosis [Rajguru and Prabhakar, (2018)]. Swarm search or Meta heuristic methods have shown better results than conventional search methods [Singhal, (2016), Fong et al., (2018), Fang et al., (2018), Yahiya, (2018), Ramasamy and Rani, (2018)]. A major problem with disease datasets is the class imbalance present in them, Class imbalance occurs when the classes of the datasets are not equally represented. In a majority of cases the positive class which represents abnormality will be the minority class and the negative class which represents normal cases will be the majority class. When classifiers are used with these datasets since the majority is the negative class the classifiers produce a moderate accuracy and the accuracy of the positive class gets overlooked in the process. This issue can be overcome by using class balancing techniques where samples can be reduced (undersampling) or samples can be increased (oversampling) or by using a combination of both [Chawla et al., (2002)]. Another issue is the misclassification of samples and it can lead to serious consequences. The performance of the classifiers can be evaluated by not only Accuracy, but with other measures such as Kappa values, Sensitivity, FPR, ROC, time to build the model and F-measure. The performance of the feature search methods vary depending on the data subsets 
selected so evaluation metrics are essential in estimating whether the selected features are optimal [Li et al., (2017)].

The aim of the study is to classify breast cancer tumors as malignant or benign using the Logistic regression model integrated with a feature search technique ant search and class balancing. The first objective of this work is to reduce the number of features in the cancer dataset using ant search, and the second objective is to classify the cancer as benign or malignant using Logistic Regression and evaluate the classifier using various performance measures. To compare the performance of the proposed model, it is evaluated against four other classification techniques Naïve Bayes, Neural Networks, Random Forest, and Support Vector Machines.

The rest of this paper is organized as follows. In Section 2, the related work on the classification of diseases using various Meta heuristic algorithms is reviewed. In Section 3, the implementation of our feature selection strategy with class balancing techniques is described. The evaluation of the proposed method is discussed and analyzed in Section 4. Section 5 makes a conclusion of the study done.

\section{Related Works}

To improve classification of incomplete data Tran et al., (2017) proposed a combination of bagging and feature selection methods. The C4.5 and REPTree classifiers were used with Particle Swam Optimization as the search technique for feature selection and achieved an accuracy of $96.19 \%$ and $96.03 \%$ respectively for the breast cancer dataset. Rajendran et al., (2020) in their work using the combination of SMOTE and spreadsubsample with Naïve Bayes, Bayesian Networks, Random Forest and Decision tree C 4.5 classifier and they compared performance of the classifiers. Bayesian network was seen to provide an overall better performance with an ROC of 0.937 . Reddy and Khare, (2017) introduced the FFBAT-ANN prediction algorithm for Diabetes disease classification and the results outperformed conventional methods. Mazen et al., (2016) proposed Genetic Algorithm based Firefly Algorithm approach to classify Breast cancer and it was capable of achieving the lowest mean squared error of 0.0014. Emami and Pakzad, (2019) proposed an approach, of two phases. In the first phase, the Affinity Propagation (AP) clustering method was used for instance reduction, by finding noisy instances and eliminating them. In the second phase, feature selection and classification was conducted by using the Adaptive Modified Binary Firefly Algorithm using SVM. A classification accuracy of $98.606 \%$ was obtained. Nadira and Rustam, (2017) proposed a Global Artificial Bee Colony - Support Vector Machine for classification of various cancers. The results showed classification accuracy on breast cancer dataset of $96.4286 \%$. Saoud et al., (2019) proposed an LR model with Best First search algorithm for Breast cancer classification and obtained an accuracy of 96.70\%.Dhahri et al., (2020) proposed an LR model with Tabu Search and obtained an accuracy of $98 \%$ on the WDBC dataset. Mathew, (2019b) in her work used Logistic regression with a greedy feature seelction algorithm RFE to select relevant features to classify Breast Cancer. The model gave an accuracy of $95.98 \%$.

\section{Methodology and Techniques}

The methodology involves selection of the dataset, preprocessing it by initially removing instances with missing values and then transforming it into nominal values. This data is fed to the machine learning techniques and performance is evaluated. Feature selection is done and few attributes are selected and this refined dataset is also run on each classifier to see the results. On the training dataset, balancing techniques are applied. The dataset is then fed to, the Logistic regression classifier and the performance of is evaluated using various evaluation measures. On the balanced dataset feature selection is applied and then this is passed to the classifier and performance is evaluated. The search algorithms reduced the number of attributes by avoiding irrelevant features. The performance measures used are Accuracy, ROC, FPR, Recall, F measure. 10 fold cross validation is done. Fig 1 shows the working of the proposed model.

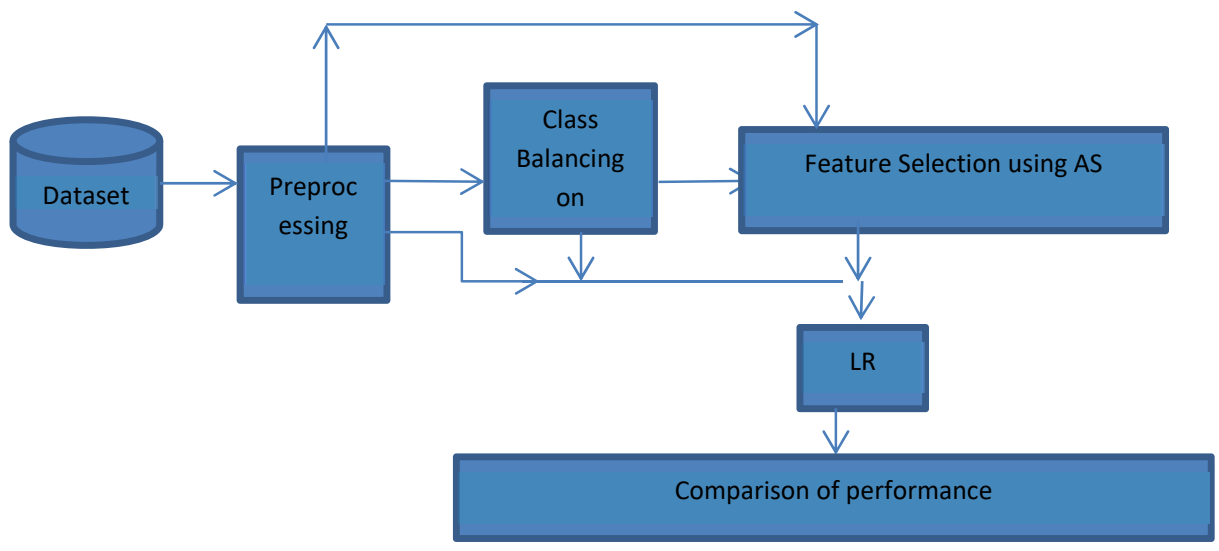

Fig 1: Working of the model 


\subsection{Data Mining Techniques Used}

\subsubsection{Logistic Regression}

Logistic Regression is a supervised classifier that models data using a sigmoid function. The advantage of using LR is that it maximizes the quality of output on a training set and makes no assumptions on the distribution of classes in the feature space. A major problem with the classifier is that it is insensitive to imbalanced data and outliers. The model provides the relationship between one dependent binary variable and the many independent variables. The probability that a data point belongs to a specific class is given by the Logistic model. For a dataset with $\mathrm{n}$ features and $\mathrm{p}$ instances the feature matric $\mathrm{X}=\left[\begin{array}{cccc}1 x 11 & x 12 & \cdots & x 1 n \\ \vdots & \ddots & \vdots \\ 1 x p 1 & x p 2 & \cdots & x p n\end{array}\right]$, where $\mathrm{x}_{\mathrm{ij}}$ represent the $\mathrm{j}^{\text {th }}$ feature of the $i^{\text {th }}$ instance.

The sigmoid function is used as objective function and the aim is to minimize it. The logistic function "Eq.( 1)" is given as

$$
\varnothing(\mathrm{z})=\frac{1}{1-e^{-z}}
$$

$\varnothing(z)$ is always bounded within $(0,1)$. The net input function, $z$, is a dot product of the input features and the respective regression coefficients and is denoted "Eq. (2)" as

$$
\begin{aligned}
& \mathrm{z}=\mathrm{x}_{0} \mathrm{w}_{\mathrm{o}}+\mathrm{x}_{1} \mathrm{w}_{1}+\ldots . \mathrm{x}_{\mathrm{n}} \mathrm{W}_{\mathrm{n}}=\sum_{j=1}^{n} \mathrm{wjxj}=\mathrm{wTx} \\
& \mathrm{z} \text { can also be represented as } \operatorname{logit}(\mathrm{p}(\mathrm{y}=1 \mid \mathrm{x}),
\end{aligned}
$$

Where $\mathrm{p}(\mathrm{y}=1 \mid \mathrm{x})$ is the conditional probability that data point belongs to class 1 given its features $\mathrm{x}$. This is inverse to the logistic function and once model fitting is done, the conditional probability $\mathrm{p}(\mathrm{y}=1 \mid \mathrm{x})$ is transformed to a binary class label thru $\mathrm{g}(\mathrm{z})$, a threshold function "Eq.(4)" and

$$
\begin{gathered}
\mathrm{g}(\mathrm{z})=1 \text { if } \varnothing(\mathrm{z}) \geq 0.5 \\
=0 \text { else }
\end{gathered}
$$

To minimize the logistic function the Maximum likelihood function is used. The log likelihood function is maximized or alternatively a cost function can be defined to be minimized. This cost function "Eq. (5)" can be defined as

$$
\begin{array}{r}
H(w)=-\log (\varnothing(z)) \text { if } y=1 \\
=-\log (1-\varnothing(z)) \text { if } y=0
\end{array}
$$

To prevent overfitting L2 parameter regularization is done. Large weight values are penalized to reduce the model complexity. The regularization term is added to the cost function. The L2 parameter "Eq. (6)" is given as

$$
\mathrm{L} 2=\frac{\wedge}{2} \sum w_{j} 2, \mathrm{j}=1 \ldots \mathrm{n}
$$

\subsection{Class Balancing Techniques}

\subsubsection{Oversampling using SMOTE (OS)}

Synthetic minority oversampling technique (SMOTE) is an oversampling method used for class imbalance problems. In SMOTE minority class examples are randomly increased by replicating them. To be precise, new minority instances are synthesized between the existing minority instances. The synthetic records are generated by the random selection of $\mathrm{k}$ nearest neighbours of the instances in the minority class.

\subsubsection{Undersampling using Random Subsampling (US)}

Random Undersampling involves randomly selecting examples from the majority class to be removed from the training dataset. To be specific, a sample down procedure is done on the majority class data until it occurs with the same frequency as the minority class The major limitation of Undersampling is that instances from the majority class that are deleted may be useful important information or even perhaps critical to fitting a robust decision boundary and this can influence the performance of the model.

\subsubsection{Combination of Oversampling and Undersampling (OS+US)}

A moderate increase in minority class instances and moderate reduction in majority class instances help in improving and reducing the bias involved in the two situations. First the difference between the majority and minority class samples are calculated. Then the number of samples to be removed from the majority class and number of samples to be increased for the minority class are determined. Then, the majority class samples are reduced and the minority class samples are increased accordingly. 


\subsection{Feature Search Techniques}

\subsubsection{Ant Search (AntSrch/AS)}

Ant Search is based on the Ant Colony optimization technique proposed by Marco Dorigo and colleagues in 1990s. It is a population based metaheuristic technique. It is inspired by the foraging behavior of ants seeking an optimal path from the food source to their colony. Ants live and work in colonies and as a group exhibit highly organized capabilities. They travel the shortest path between their food sources and nest. They communicate with each other through pheromones as they have low visibility. Pheromones are chemical substances released while an ant travels on the ground. These mark trails on the ground and other ants follow this path. The collective behavior of ants is used as an optimization tool. Initially ants move in random searching for food. Hence multiple paths are created. A portion of food is carried back to the nest if the quantity and quality are right, and they leave pheromone trails on the way back. This acts as a guide to other ants. Pheromone evaporation is also to be taken into account. On the less travelled trail the pheromones evaporate and hence the most frequently travelled path will have a high intensity of pheromones. The intensity of pheromones on the travelled path increases as each ant traversing it deposits pheromones on it. There are various variants to the original ACO algorithm. Elitist ant systems, Ant colony system, Max- Min ant system, rank based ant systems, and continuous orthogonal ant systems,

The algorithm for ant search is given as

Procedure AntSearch()

Initialize pheromone trails and parameters.- population size $n$, maximum iterations, pheromone value, fitness value, pheromone evaporation rate $T$,

While (not terminated)

Generate ant population

Calculate fitness value for each ant

Find best solution through roulette wheel selection criteria

Update pheromone trail.

End while

Display best ant(solution), best fitness value

End

\subsection{Data Used}

The benchmark Breast Cancer datasets of the Wisconsin Hospitals from the UCI repository is being used. This breast cancer databases was obtained from the University of Wisconsin Hospitals, Madison from Dr. William H. Wolberg. The Breast Cancer Wisconsin Original data Set has 699 instances, 11 attributes and 13 missing instances with 458 benign (65.5\%) and $241(34.5 \%)$ malignant cases. The data features are computed from the digitized image of a fine needle aspirate (FNA) of a breast mass and describe characteristics of the cell nuclei present in the image. The attributes are shown in Table 1. Id Number, since it has no relevance in the classification process is discarded from the set of attributes.

TABLE 1 Attributes

\begin{tabular}{|c|l|c|c|c|}
\hline \multirow{2}{*}{ Number } & Attribute Name & \multirow{2}{*}{ Values } & \multicolumn{2}{|c|}{ Comparison of malignant and benign cells } \\
\cline { 4 - 5 } & & & Malignant & Benign \\
\hline 1 & Clump_thickness & $1-10$ & Seen in Multilayers & Seen in monolayers \\
\hline 2 & Size_uniformity & $1-10$ & Size differs & Unifrom size \\
\hline 3 & Shape_uniformity & $1-10$ & Shape differs & Unifrom Shape \\
\hline 4 & Marginal_adhesion & $1-10$ & Cells don not stick together & Cells stick together \\
\hline 5 & Epithelial_size & $1-10$ & Enlarged & Small \\
\hline 6 & Bare_nucleoli & $1-10$ & Have bare Nucleoli & No Bare Nucleoli \\
\hline 7 & Bland_chromatin & $1-10$ & Coarse in texture & Uniform texture \\
\hline 8 & Normal_nucleoli & $1-10$ & Nucleus is bigger & Nucleus is small \\
\hline 9 & Mitosis & $1-10$ & More Mitosis & Not so \\
\hline 10 & Class & $\begin{array}{c}\text { 2-Benign 4- } \\
\text { Malignant }\end{array}$ & & \\
\hline
\end{tabular}




\subsection{Evaluation Metrics used}

Various metrics are available for evaluation of models. The metrics used for evaluation of the model in this study are accuracy, ROC, Mathews Correlation Coefficient (MCC), Kappa Statistic, Precision and Recall.

\subsubsection{Accuracy}

Accuracy is the number of correct classifications made by the model. It is evaluated as

$$
\text { Accuracy }=\frac{T P+T N}{T P+T N+F P+F N} \times 100
$$

TP, TN, FP, FN being the True Positives, True Negative, False Positives and False Negatives obtained from the confusion matrix.

3.5.2 Mathews Correlation Coefficient

\subsubsection{F Score}

$$
\mathrm{MCC}=\frac{=T P x T N-F P x F N}{\sqrt{(T P+F P)(T P+F N)(T N+F P)(T N+F N)}}
$$

F score is the harmonic mean of Precision and Recall

$$
\text { F score }=\frac{2 T P}{2 T P+F P+F N}
$$

\subsubsection{Kappa Statistic}

It compares the expected and observed outcome and is given by

$$
\text { Kappa }=\frac{\text { total accuracy }- \text { random accuracy }}{1-\text { random accuracy }}
$$

And random accuracy $=\frac{(T N+F P)(T N+F N)+(F N+T P)(F P+T P)}{\text { total } x \text { total }}$

And total accuracy $=$ Accuracy

\subsubsection{Recall}

Recall is also known as sensitivity gives the number of correctly classified true positives.

$$
\text { Recall }=\frac{T P}{T P+F N}
$$

\subsubsection{Precision}

Precision gives the number of true positives against the number of positives identified.

$$
\text { Precision }=\frac{T P}{T P+F P}
$$

\section{Results and Discussion}

The performance of the hybrid model obtained is shown in Table 2. Accuracy "Eq. (7)" of $99.4 \%$ was attained by the proposed model. MCC "Eq. (8) is a reliable score which produces a good result only if a good prediction score is arrived for the four categories of the confusion matrix. It shows how well the classifier performs and the proposed model displayed it at 0.988 . Table 2 gives the comparison of the various cases of accuracy of the classifier. ROC, Fig 2 depicts the tradeoff between TPR and FPR. The ROC value of 0.998 was obtained and in figure 2 it can be seen along the $y$ axis at leftmost edge of the graph. The Confusion matrix presents the correctly classified and misclassified instances of the two classes. The hybrid model was seen to classify the positive classes correctly except for one instance and 3 wrongly classified instance for the negative class. The kappa statistic "Eq. (10)" measures the interrater reliability viz. expected and observed outcomes. A value of 1 shows perfect agreement. The proposed model achieved a good value of 0.9883. The F measure "Eq.(9)" is obtained as the harmonic mean of precision and recall. Recall "Eq. (11)" gives the ratio of correctly predicted positive observations to the all observations in actual class. The proposed model achieved a recall, precision "Eq. (12)" and F measure of 0.994 each.

The proposed model is compared with the conventional Logistic Regression classifier, the LR classifier with Oversampling alone performed, LR classifier with Undersampling alone done and with feature search performed with the LR classifier. The proposed model outperformed them in all the cases. Oversampling improved the accuracy measure considerably when compared with the technique with no class balancing. This is due to the increase in samples of the minority class. The performance of Undersampling was reduced and seen as the least efficient among the class balancing methods applied in the models. This is due to the loss of useful information when samples are reduced from the majority class. The combination of Undersampling and Oversampling produced the best results with the Logistic Regression classifier. This was similar with the four other classifiers used for comparison, (Table 3). Table 2 shows the various performance metrics used. In every case the proposed hybrid model performed comparatively better than them all. 
Table 2 Performance Metrics

\begin{tabular}{|c|c|c|c|c|c|c|c|c|c|c|}
\hline Classifiers & Accuracy & Kappa & $\mathrm{ROC}$ & FPR & $\begin{array}{c}\text { F- } \\
\text { Measure }\end{array}$ & $\mathrm{MCC}$ & Recall & Precision & $\begin{array}{l}\text { Time taken } \\
\text { to build } \\
\text { model (secs) }\end{array}$ & $\begin{array}{c}\text { Confusion } \\
\text { Matrix }\end{array}$ \\
\hline LR & 92.53 & 0.8321 & 0.928 & 0.112 & 0.924 & 0.835 & 0.925 & 0.926 & 0.06 & $\begin{array}{l}430 \quad 14 \\
37202\end{array}$ \\
\hline $\mathrm{LR}+\mathrm{OS}$ & 96.42 & 0.9284 & 0.988 & 0.035 & 0.964 & 0.929 & 0.964 & 0.965 & 0.28 & $\begin{array}{ll}433 & 11 \\
22 & 456\end{array}$ \\
\hline $\mathrm{LR}+\mathrm{US}$ & 92.25 & 0.8452 & 0.958 & 0.077 & 0.923 & 0.846 & 0.923 & 0.924 & 0.16 & \\
\hline $\mathrm{LR}+(\mathrm{OS}+\mathrm{US})$ & 99.1 & 0.9824 & 0.999 & 0.009 & 0.991 & 0.982 & 0.991 & 0.991 & 0.05 & $\begin{array}{l}3392 \\
4337\end{array}$ \\
\hline LR+Ant Search & 91.9 & 0.8311 & 0.920 & 0.116 & 0.921 & 0.832 & 0.921 & 0.921 & 0.06 & $\begin{array}{l}41826 \\
29210\end{array}$ \\
\hline $\begin{array}{l}\text { Proposed Hybrid } \\
\text { Model- } \\
\text { LR+OS+US+An } \\
\text { tSrch }\end{array}$ & 99.4 & 0.9883 & 0.998 & 0.006 & 0.994 & 0.988 & 0.994 & 0.994 & 0.03 & $\begin{array}{cr}338 & 3 \\
1 & 340\end{array}$ \\
\hline
\end{tabular}

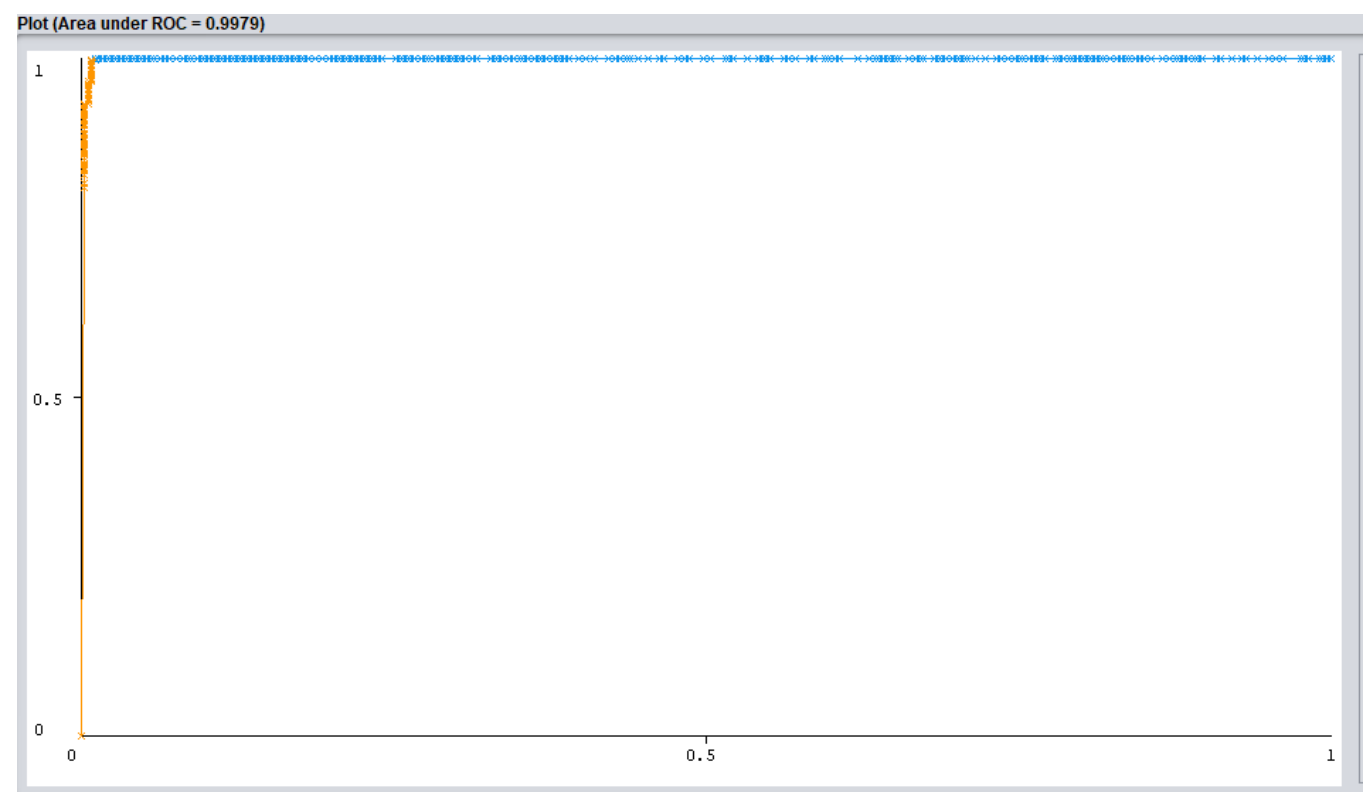

Figure 2 ROC

The performance of the proposed model is evaluated other four other classifiers- Support Vector Machines, Neural Networks and Naïve Bayes is shown in Table 3. The accuracy obtained for each classifier is displayed. SVM obtained an accuracy of $98.87 \%$, Neural Network 98.97\%, Naïve Bayes 98.3\% and Random Forest 98.82\%. The results show that the proposed model outperformed them all with an accuracy of $99.4 \%$.

Table 3 Comparison with other classifiers

\begin{tabular}{|l|c|c|}
\hline Classifier & Accuracy\% & Confusion Matrix \\
\hline SVM & 98.97 & $\begin{array}{l}3383 \\
4337\end{array}$ \\
\hline Neural Networks & 98.97 & $\begin{array}{l}3383 \\
4337\end{array}$ \\
\hline Naïve Bayes & \multirow{2}{*}{98.3} & $\begin{array}{l}3329 \\
2339\end{array}$ \\
\hline Random Forest & \multirow{2}{*}{98.82} & $\begin{array}{l}3374 \\
4337\end{array}$ \\
& & 3383 \\
& \multirow{2}{*}{99.4} & 1340 \\
\hline
\end{tabular}




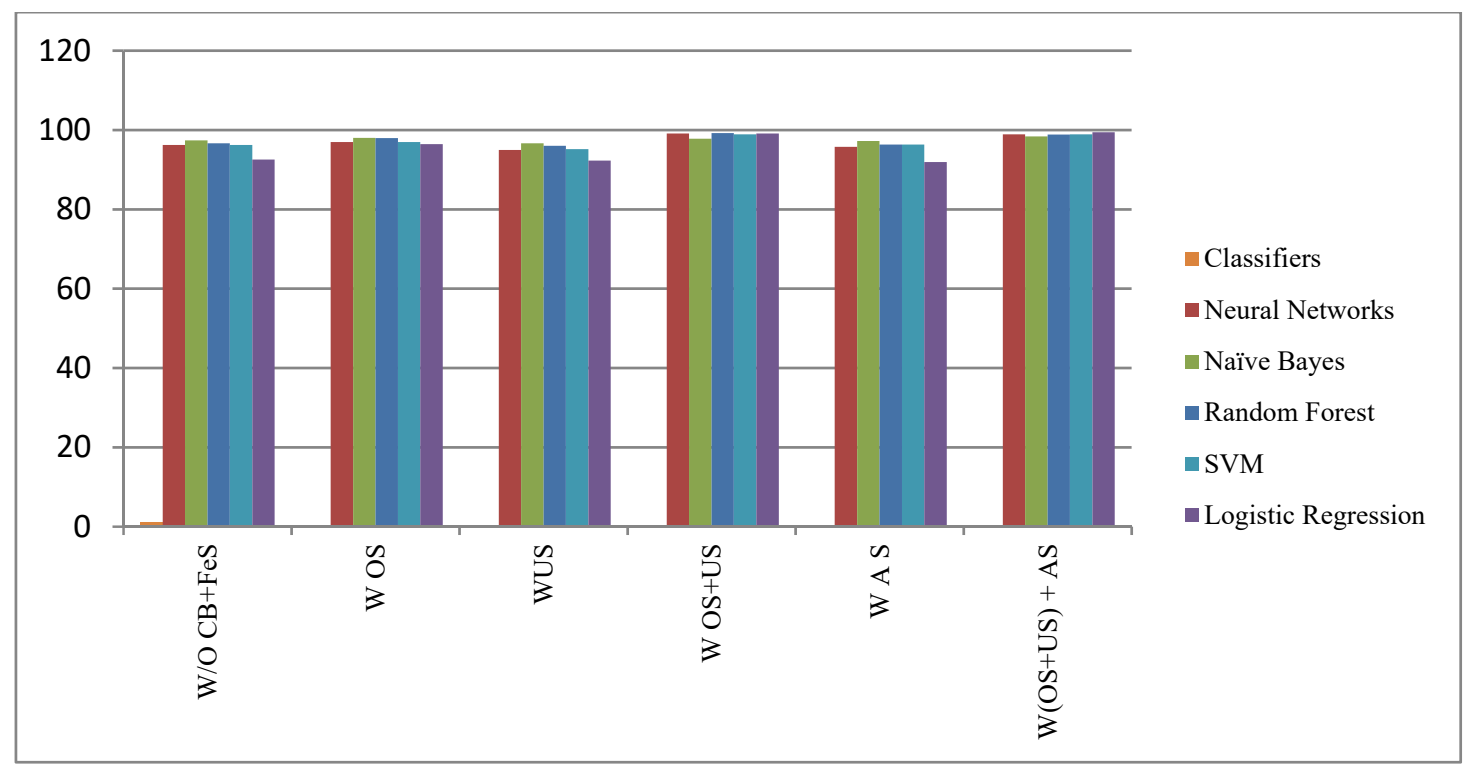

Fig 3 Comparison of Models against accuracy

In Fig 3, the performance of Logistic Regression models is presented alongwith that of the other four classifiers. The models using the classifiers alone performed comparatively lesser. The best accuracy among them was given by Naïve Bayes. With Oversampling alone Random Forest and Naïve Bayes models produced the best result. With Undersampling alone Naïve Bayes was the best classifier. With the combination of Oversampling and Undersampling performance of Logistic Regression improved considerably. Similar was the case with Random Forest, NN, and SVM, while Naïve Bayes showed a moderate performance. The best performance was by the proposed Logistic Regression and Ant search model. With Class balancing the results of all the classifiers increased considerably. With Ant search alone the best result was given by Naïve Bayes and the combination of OS+US increased the performance of all the classifiers significantly. Logistic regression improved with the combination class balancing methods and ant search techniques. Logistic regression classifier is sensitive to class imbalance. Hence applying a combination of oversampling and undersampling brought a significant accuracy increase. Combining ant search with this further increased the performance of the model. The proposed models are compared with a few other works that used swarm intelligence methods with classification techniques in Table 4

Table 4 Comparisons with other Related Works using various Search Techniques

\begin{tabular}{|l|c|c|c|}
\hline \multicolumn{1}{|c|}{ Literature } & Search Technique & Data Mining technique & Accuracy \% \\
\hline Saoud et al. (2019) [25] & Best First & LR & 96.7096 \\
\hline Dhahri et al. (2020) [3] & Tabu Search & LR & 98 \\
\hline Mathew (2019)[13] & RFE & LR & 95.98 \\
\hline Proposed work & Ant search & LR & 99.4 \\
\hline
\end{tabular}

The proposed model is compared against related works in Logistic Regression with various feature selection methods. The proposed model outperformed all in terms of accuracy.

\section{Conclusion}

The paper produced a hybrid model for breast Cancer classification using Logistic Regression on the WBCD dataset using ant search and class balancing techniques. The model was compared against four other Meta heuristic methods for attribute selection and reduction and four other classifiers alongwith three class balancing methods. Among the balancing methods used combination of Undersampling and Oversampling was seen most effective in Logistic Regression. Feature selection using Ant Search, when applied on the model made improvement to the various performance measures. The best accuracy measure was obtained by the hybrid Logistic Regression model using the combination of class balancing and Ant Search methods with a value of $99.4 \%$. Ensemble methods can be devised so as to improve the performance of other feature selection methods with logistic Regression for classification in two class problems. Modification of the cost function and application of optimization techniques for improving the Logistic regression model can also be explored 


\section{Acknowledgments}

We are indebted to Dr William H Wolberg of the University of Wisconsin Hospitals, Madison for the breast cancer databases made available.

\section{References}

[1] Arafat, H, Barakat, S, Goweda, A F, Using Intelligent Techniques for Breast Cancer Classification, International Journal of Emerging Trends \& Technology in Computer Science Volume I, Issue 3, September-October 2012

[2] Chawla, N V, Bowyer, K W , Hall, L, O, Kegelmeyer, W P, SMOTE: Synthetic Minority Oversampling Technique, Journal of Artificial Intelligence Research 16 (2002),321-357

[3] Dhahri, H, Mahmood,R A, Maghayreh, E A, and Elkilani, W Tabu Search and Machine-Learning Classification of Benign and Malignant Proliferative Breast Lesions, BioMed research International, Volume 2020, Article ID 4671349

[4] Deb, S, Fong,S, Tian, Z, Elephant Search Algorithm for Optimization Problems, The Tenth International Conference on Digital Information Management (ICDIM 2015)

[5] Durga, T S, Assiri, A S, Nazir,S, Velastin,S A, breast Tumor Classification using an Ensemble Machine Learning Method, J Imaging, $2020,6,39$

[6] Emami, N A. Pakzad, A New Knowledge-based System for Diagnosis of Breast Cancer by a combination of Affinity Propagation Clustering and Firefly Algorithm, Journal of AI and Data Mining Vol 7, No 1, 2019, 59-68

[7] Fang, M, Lei, X, Cheng, S, Shi, Y, Wu, F X, Feature Selection via Swarm Intelligence for Determining Protein Essentiality, Molecules 2018, 23, 1569

[8] Fong, S Biuk-Aghai, R P, RicMillham ,R C, Swarm Search Methods in Weka for Data Mining, ICMLC 2018: Proceedings of the 2018 10th International Conference on Machine Learning and Computing, February 2018 Pages 122-127

[9] Jabbar, S F, A classification model on tumor cancer disease based mutual information and firefly algorithm, Periodicals of Engineering and Natural Sciences ISSN 2303-4521 Vol. 7, No. 3, September 2019, pp.1152-1162

[10] Kotsiantis, S B, “Supervised machine learning: a review of classification techniques,” Informatica, vol. 31, no. 3, pp. 249-268, 2007.

[11] Li, J, Fong, S, Wong, R K, Millham, R,. Wong, K K L, Elitist Binary Wolf Search Algorithm for Heuristic Feature Selection in HighDimensional Bioinformatics Datasets, Scientific Reports volume 7, Article number: 4354 (2017)

[12] Mathew T.E, A comparative study of the performance of different Support Vector machine Kernels in Breast Cancer Diagnosis, International Journal of Information and Computing Science, Volume 6, Issue 6, pp. 432-441 June 2019

[13] Mathew T E, A Logistic Regression with Recursive Feature Elimination Model for Breast Cancer Diagnosis, International Journal on Emerging Technologies 10(3): 55-63(2019)

[14] Mathew T E, Simple and Ensemble Decision tree Classifier based detection of Breast Cancer, International Journal of Scientific \& Technology Research Volume 8, Issue 11, pp. 1628-1637, November 2019

[15] Mazen, F, AbulSeoud, R A, Gody ,A M, Genetic Algorithm and Firefly Algorithm in a Hybrid Approach for Breast Cancer Diagnosis, International Journal of Computer Trends and Technology (IJCTT) - Volume 32 Number 2 - February 2016

[16] Nadira,T , Rustam, Z, Classification of cancer data using support vector machines with features selection method based on global artificial bee colony, Proceedings of the 3rd International Symposium on Current Progress in Mathematics and Sciences 2017 (ISCPMS2017)AIP Conference Proceedings 2023, 020205 (2018),pp 1-7

[17] Rahman, M, M A, , Muniyandi, R C, An Enhancement in Cancer Classification Accuracy Using a Two-Step Feature Selection Method Based on Artificial Neural Networks with 15 Neurons, Symmetry 2020, 12, 271, 2- 21

[18] Rajaguru H, Prabhakar, S K, A Study on Firefly Algorithm for Breast Cancer Classification, Lecture Notes in Computational Vision and Biomechanics Volume 30, 2018, 421-428

[19] Rajalaxmi,R R, E.Gothai, .R.Thamilselvan, P.G Bindha, P.Natesan, Naïve Bayes guided Binary Firefly Algorithm for Gene Selection in Cancer Classification, International Journal of recent technology and engineering, Volume 8, issue 4, November 2019

[20] Rajendran, K, Jayabalan, M , Thiruchelvam, V , Predicting Breast Cancer via Supervised Machine Learning Methods on Class Imbalanced Data, (IJACSA) International Journal of Advanced Computer Science and Applications, Vol. 11, No. 8, 2020

[21] Ramasamy, R and Rani, S, Modified Binary Bat Algorithm for Feature Selection in Unsupervised Learning, The International Arab Journal of Information Technology, Vol. 15, No. 6, November 2018

[22] Rania, R R , .Ramyachitra,D, Microarray Cancer Gene Feature Selection Using Spider Monkey Optimization Algorithm and Cancer Classification using SVM, Procedia Computer Science 143 (2018) 108-116

[23] Reddy, G T, Khare, N, Hybrid Firefly-Bat Optimized Fuzzy Artificial Neural Network Based Classifier for Diabetes Diagnosis, International Journal of Intelligent Engineering and Systems, Vol.10, No.4, 2017

[24] Sadeghipour, E Sahragard, N, Sayebani,M R, Mahdizadeh,R, Breast Cancer Detection Based On A Hybrid Approach Of Firefly Algorithm And Intelligent Systems, Indian Journal of Fundamental and Applied Life Sciences , 2015, 468-472

[25] Saoud, H, Ghadi,A ,Ghailani, M, Abdelhakim, B A, Using Feature Selection Techniques to Improve the Accuracy of Breast Cancer Classification, (C) Springer Nature Switzerland AG 2019 M. Ben Ahmed et al. (Eds.): SCA 2018, LNITI, pp. 307-315, 2019.

[26] Singhal, S K, An Evolutionary Bayesian Network Learning Algorithm using Feature Subset Selection for Bayesian Network Classifiers, International Journal of Computer Applications (0975 - 8887) Volume 135 - No.13, February 2016

[27] Sivapriya,T R, A. R. Kamal, N B, Thangaiah,, P R J, Ensemble Merit Merge Feature Selection for Enhanced Multinomial Classification in Alzheimer's Dementia, Computational and Mathematical Methods in Medicine Volume 2015, Article ID 676129, 11 pages

[28] Tran, C T, Zhang, M, Andreae, P, and Xue,B, Bagging and Feature Selection for Classification with Incomplete Data, Conference: Evostar, April 2017, 471- 486

[29] Yahya, A A, Centroid particle swarm optimisation for highdimensional data classification, Journal Of Experimental \& Theoretical Artificial Intelligence, 2018 